0.4 prescription per individual) for GC during the study period. GC use varied between patient groups (Figure 1). The total GC use (DDD) doubled over the two-year period leading up to TNFi treatment but decreased sharply after the initiation of TNFi. The number of individuals on GC decreased by one third after initiating TNFi therapy and the majority of those who continued GC treatment were patients with RA (Figure 1). Of those patients on long term GC treatment $(>7.5 \mathrm{mg} /$ day for three months) $38 \%$ were receiving bone protective therapy against corticosteroid induced osteoporosis. The use of topical steroids decreased by half among PsA patients and one third discontinued the treatment after initiating TNFi (Figure 2).

Conclusion: TNFi therapy does impact GC use among patients with arthritides, however a large portion of RA patients are still on GC two years after initiating TNFi therapy. Better osteoporosis prophylaxis and treatment is warranted for those patients on long term GC

Figure 1: Percentage of patients that received glucocorticosteroids

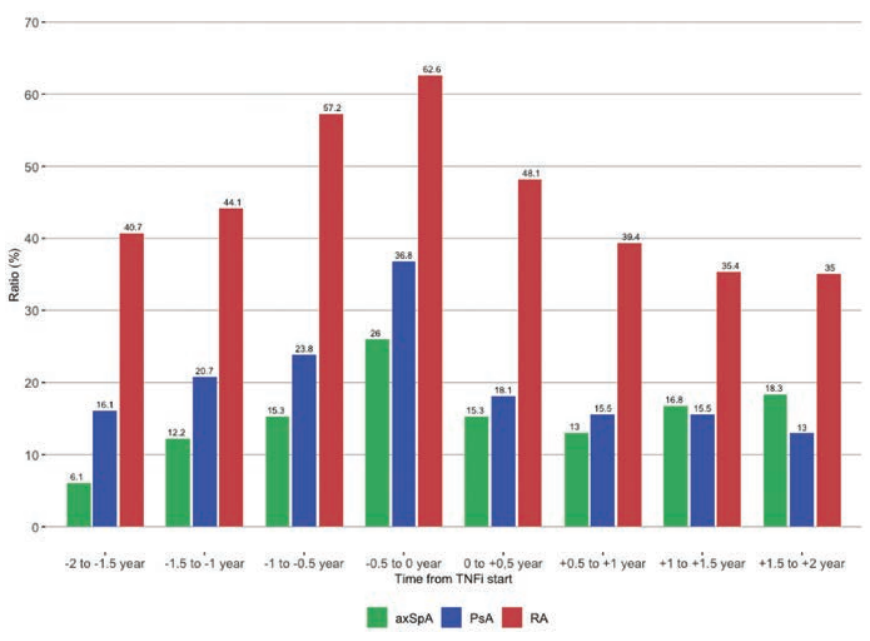

Figure 2: Total number of prescriptions on topical corticosteroids in PsA

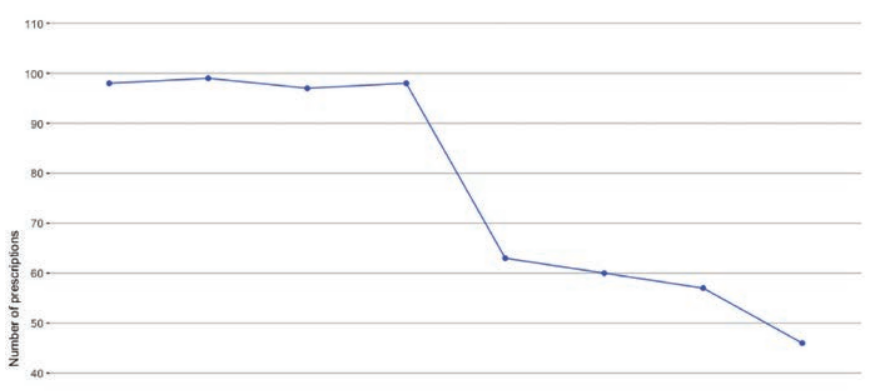

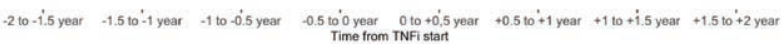

Disclosure of Interests: Rebekka Hafthorsdottir: None declared, Anna Gunnarsdottir: None declared, Thorvardur Love: None declared, Gerdur Gröndal: None declared, Björn Gudbjornsson Speakers bureau: Novartis and Amgen DOI: 10.1136/annrheumdis-2020-eular.2103

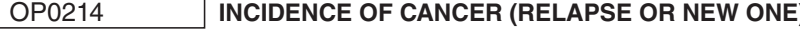 FOLLOWING BDMARDS INITIATION IN PATIENT WITH RHEUMATOID ARTHRITIS AND HISTORY OF CANCER: A SYSTEMATIC REVIEW WITH META-ANALYSIS OF OBSERVATIONAL STUDIES}

A. Wetzman ${ }^{1}$, T. Barnetche ${ }^{2}$, C. Lukas ${ }^{1}$, C. Gaujoux-Viala ${ }^{3}$, B. Combe ${ }^{1}$, J. Morel ${ }^{1}$, P. Szafors ${ }^{1} .{ }^{1} \mathrm{CHU}$ Lapeyronie, Department of Rheumatology, Montpellier, France; ${ }^{2} \mathrm{CHU}$ Pellegrin, Bordeaux, France; ${ }^{3} \mathrm{CHU}$ Nïmes, Department of Rheumatology, Nimes, France
Background: The tolerance of targeted biologic therapies (bDMARDs) used in rheumatoid arthritis (RA) has been studied in patients with no history of cancer. A 5-year cancer remission is recommended to initiate treatment with bDMARDs. However, in the context of the aging of the population and the increase in screening for neoplasia, the question of targeted treatment in a patient with RA with a history of cancer is usual.

Objectives: To determine the risk of recurrence or a new malignancy when exposed to a b-DMARDs in adults with RA and a history of cancer.

Methods: A systematic literature search of EMBASE, MEDLINE, Cochrane Library, and electronic abstract databases of the annual scientific meetings of both the European League Against Rheumatism and the American College of Rheumatology was conducted through June 2019 and selected all cohort follow-up studies including adults with RA with a history of cancer and treated with b-DMARDs (TNF inhibitors, rituximab, abatacept and tocilizumab). We compared the risk of relapse or new cancer onset between the groups treated with and without b-DMARDs. The RevMan 5.3 software was used to calculate the cumulative risks from each group's Hazard Ratio (HR), with their $95 \%$ confidence intervals. The heterogeneity of the studies was evaluated by the Cochran $Q$ test and expressed with the $\mathrm{I}^{2}$ value.

Results: 26 observational cohort studies were selected, of which 12 were included in the meta-analysis. The overall risk of new cancer or recurrence of neoplasia in RA patients with a history of cancer, compared to control subjects, for all b-DMARDs combined was $1.09\left(p=0.29,95 \% \mathrm{Cl}[0.92-1.32], \mathrm{I}^{2}=16 \%\right)$. The risk of relapse or new cancer onset in patients exposed to TNF inhibitors was $1.11\left(p=0.45,95 \% \mathrm{Cl}[0.85-1.46], \mathrm{I}^{2}=48 \%\right)$ and $0.79(\mathrm{p}=0.49,95 \% \mathrm{Cl}[0.41-1.53]$, $\left.\mathrm{I}^{2}=10 \%\right)$ for rituximab. The rate of recurrence was $1.32(\mathrm{p}=0.04,95 \% \mathrm{Cl}[1.02$ 1.72], $\left.I^{2}=0 \%\right)$ for skin cancer, $1.28\left(\mathrm{p}=0.07,95 \% \mathrm{Cl}[0.98-1.67], \mathrm{I}^{2}=0 \%\right)$ for skin cancers excluding melanoma and $1.21\left(\mathrm{p}=0.31,95 \% \mathrm{Cl}[0.84-1.72], \mathrm{I}^{2}=0 \%\right)$ for breast neoplasia.

Conclusion: This is the first meta-analysis evaluating the risk of recurrence or new cancers in a RA population with a history of neoplasia exposed to b-DMARD. For patients with a history of cancer, for whom the benefit / risk balance has been found to be favorable, the initiation of b-DMARD treatment for RA does not seem to significantly increase the risk of recurrence or new cancer compared to patients naïve to biological treatments, apart from skin cancers including melanoma.

Disclosure of Interests: Amélie Wetzman: None declared, Thomas Barnetche: None declared, Cédric Lukas: None declared, Cecile Gaujoux-Viala: None declared, Bernard Combe Grant/research support from: Novartis, Pfizer, Roche-Chugai, Consultant of: AbbVie; Gilead Sciences, Inc.; Janssen; Eli Lilly and Company; Pfizer; Roche-Chugai; Sanofi, Speakers bureau: Bristol-Myers Squibb; Gilead Sciences, Inc.; Eli Lilly and Company; Merck Sharp \& Dohme; Pfizer; Roche-Chugai; UCB, Jacques Morel: None declared, Paulina Szafors: None declared DOI: 10.1136/annrheumdis-2020-eular.4963

\section{OP0215 FUNCTIONAL ABILITIES IMPROVEMENT IN RHEUMATOID ARTHRITIS PATIENTS WITH DEPRESSIVE AND ANXIETY DISORDERS TREATED WITH BIOLOGIC DMARDS AND/OR ANTIDEPRESSANTS}

A. Abramkin ${ }^{1}$, T. Lisitsyna ${ }^{1}$, D. Veltishchev ${ }^{2,3}$, O. Seravina ${ }^{2}$, O. Kovalevskaya ${ }^{2}$ E. Nasonov ${ }^{1,4}{ }^{1}$ V.A. Nasonova Research Institute of Rheumatology, Vascular Rheumatology, Moscow, Russian Federation; ${ }^{2}$ Moscow Research Institute of Psychiatry, National Medical Research Center of Psychiatry and Narcology, Ministry of Health of Russia, Stress Disorders, Moscow, Russian Federation; ${ }^{3}$ N.I. Pirogov Russian Research Medical University, Ministry of Health of Russia, Psychiatry, Moscow, Russian Federation; ${ }^{4}$ Institute of Professional Education, I.M. Sechenov First Moscow State Medical University (Sechenov University), Ministry of Health of Russia, Rheumatology, Moscow, Russian Federation

Background: Anxiety and depressive disorders (ADD) significantly increase functional limitations in patients with rheumatoid arthritis (RA). Successful psychopharmacotherapy (PPT) of ADD can potentially improve the functional abilities of RA-patients.

Objectives: To compare changes in functional disability of RA patients with comorbid ADD treated with conventional disease-modifying antirheumatic drugs (CDMARDs) alone or in combination with biologic DMARDs (bDMARDs) and/or PPT.

Methods: 128 RA-patients (pts) were enrolled, $86 \%$ were women with a mean age of $47,4 \pm 11,3(\mathrm{M} \pm \mathrm{SD})$ yrs. All patients met the full ACR/EULAR 2010 criteria for RA. Functional limitations were assessed using Health Assessment Questionnaire (HAQ), mean HAQ was 1,42 $\pm 0,78$ at baseline. $69,4 \%$ RA-pts were already taking prednisone (9 $[5 ; 10] \mathrm{mg} /$ day (Me $(25 \% ; 75 \%)), 84,4 \%$ cDMARDs, $7,8 \%$ - bDMARDs (anti-TNF- $a-6,3 \%$, rituximab - 1,6\%). ADD were diagnosed by psychiatrist in $123(96,1 \%)$ of RA-pts in accordance with ICD-10 in semi-structured interview. Severity of depression and anxiety was evaluated with 
Montgomery-Asberg Depression Rating Scale (MADRS) and Hamilton Anxiety Rating Scale (HAM-A). RA-pts with ADD were divided into the following treatment groups: 1 - cDMARDs ( $n=39), 2-$ cDMARDs + PPT (sertraline or mianserine) $(n=43), 3-c D M A R D s+$ bDMARDs $(n=32), 4-c D M A R D s+$ bDMARDs + PPT (sertraline or mianserine) $(n=9)$. Biologics treatment duration varied from 1 to 6 years, antidepressants - from 6 to 96 weeks.

Results: $\mathrm{HAQ}$ scores were high in all 4 groups at baseline and after five years remained high in all groups except group 2 with the lowest endpoint scores among 4 groups (table 1). To measure changes in HAQ scores between groups we compared the differences between baseline and endpoint $\mathrm{HAQ}$ scores $(\triangle \mathrm{HAQ}=$ endpoint $\mathrm{HAQ}$ - baseline $\mathrm{HAQ}$ ) (table 2). The table shows an improvement in HAQ scores in groups 2 and 3 , no significant changes in group 4 and a worsening of HAQ scores in group 1. $\mathrm{HAQ}$ scores in groups 2 and 3 significantly improved compared to group 1.

Table 1 Mean HAQ scores in RA patients with ADD at baseline and after 5 years, by groups.

\begin{tabular}{lccc}
\hline Groups & $\begin{array}{c}\text { At baseline } \\
(\mathrm{n}=128)\end{array}$ & $\begin{array}{c}\text { After } 5 \text { years } \\
(\mathrm{n}=83)\end{array}$ & $\begin{array}{c}\text { P between } \\
\text { time points }\end{array}$ \\
\hline 1 (cDMARDs), $\mathrm{n}=39$ & $1,39 \pm 0,75$ & $1,61 \pm 0,7$ & $>0,05$ \\
2 (cDMARDs + PPT), $\mathrm{n}=43$ & $1,42 \pm 0,9$ & $0,85 \pm 0,66$ & 0,011 \\
3 (cDMARDs + bDMARDs), $\mathrm{n}=32$ & $1,58 \pm 0,76$ & $1,36 \pm 0,71$ & $>0,05$ \\
4 (cDMARDs + bDMARDs + PPT), $\mathrm{n}=9$ & $1,38 \pm 0,83$ & $1,49 \pm 0,26$ & $>0,05$ \\
P between groups & $>0,05$ & $\mathrm{P}_{2-1}<0,001 ;$ & \\
& & $\mathrm{P}_{2-3}^{2}=0,023$ & \\
& & $\mathrm{P}_{2-4}=0,015$ & \\
\hline
\end{tabular}

Table 2. Differences between baseline and 5-years endpoint HAQ scores, by groups

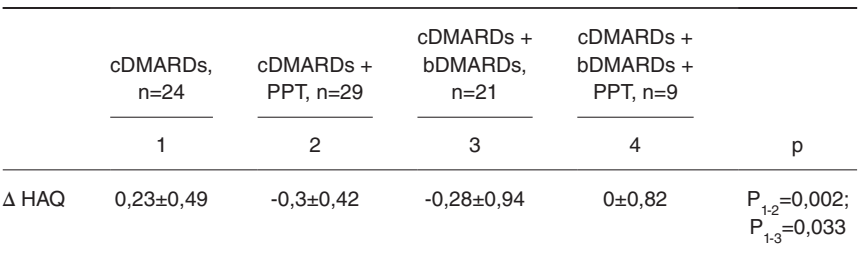

Conclusion: Functional abilities measured by HAQ scores significantly improved in RA-patients with ADD receiving cDMARDs in combination with bDMARDs or PPT compared to cDMARDs only. The lowest HAQ scores were observed in patients receiving cDMARDs in combination with PPT.

Disclosure of Interests: None declared

DOI: 10.1136/annrheumdis-2020-eular.367

\section{Rheumatoid arthritis - prognosis, predictors and outcome II}

\begin{tabular}{|l|l}
\hline OP0216 & DEVELOPMENT AND VALIDATION OF PATIENT-LEVEL \\
PREDICTION MODELS FOR ADVERSE HEALTH \\
OUTCOMES AMONGST ADULT RA PATIENTS INITIATING \\
FIRST-LINE TREATMENT OF METHOTREXATE \\
MONOTHERAPY: A MULTINATIONAL REAL-WORLD \\
COHORT ANALYSIS INCLUDING 164,735 SUBJECTS
\end{tabular}

C. Yang ${ }^{1}$, R. Williams ${ }^{2}$, J. Swerdel ${ }^{3}$, M. Jani ${ }^{4}$, T. Duarte-Salles ${ }^{5}$,

K. Chatzidionysiou ${ }^{6}$, D. Prieto-Alhambra ${ }^{7}$, P. Ryan ${ }^{8}$, P. Rijnbeek ${ }^{9}$ on behalf of 'European Health Data and Evidence Network (EHDEN) RA Research Group. ' Department of Medical Informatics EMC, Rotterdam, Netherlands; ${ }^{2}$ Department of Medical Informatics Erasmus MC, Rotterdam, Netherlands; ${ }^{3} J a n s s e n$ Research and Development, New Jersey, United States of America; ${ }^{4}$ Centre for Epidemiology Versus Arthritis, University of Manchester, Manchester, United Kingdom; ${ }^{5}$ Fundació Institut Universitari per a la Recerca a l'Atenció Primària de Salut Jordi Gol i Gurina (IDIAPJGol), Barcelona, Spain; ${ }^{6}$ Dep of Medicine, Solna, Rheum Unit, Karolinska Institutet, Stockholm, Sweden; ${ }^{7}$ University of Oxford, NDORMS, Oxford, United Kingdom; ${ }^{8}$ Johnson \& Johnson, New Jersey, United States of America; ${ }^{9}$ Erasmus University, Department of Medical Informatics, Rotterdam, Netherlands

Background: EULAR guidelines recommend the early initiation of methotrexate (MTX) monotherapy as soon as possible after the diagnosis of rheumatoid arthritis (RA). Evaluating patient-level risks for adverse outcomes after MTX initiation would allow clinicians to provide more personalised care.

Objectives: To develop and validate patient-level prediction models for adverse health outcomes including leukopenia, pancytopenia, infection (serious, opportunistic, all), cardiovascular disease (CVD) (myocardial infarction (MI), stroke), and cancer (breast, colorectal, uterus) in adult RA patients initiating first-line treatment of MTX monotherapy

Methods: Health data from claims and electronic health records were used including patients from 7 European countries (Spain, Estonia, Netherlands, Belgium, Germany, France, and the UK), the United States of America, Australia, and Japan. All RA patients initiating first-line treatment of MTX monotherapy with at least one year of prior observation were included. Prediction models for the outcomes were developed for a time at risk of 3 months (infections, leukopenia, pancytopenia), 2 years ( $\mathrm{Ml}$ and stroke), and 5 years (cancers) on the Optum $\odot$ De-Identified Clinformatics $®$ Data Mart Database. Models were developed using LASSO logistic regression and were evaluated using the area under the receiver operator characteristic curve (AUROC) for discrimination and graphically assessed for calibration. The models were externally validated on all other databases.

Results: A total of 21,307 subjects were used for training and validated against 143,427 patients from 14 sites. MI (AUROC internal 0.77, AUROC external ranging from 0.49 to 0.78 ), stroke (AUROC internal 0.78 , AUROC external ranging from 0.68 to 0.79 ) and serious infection (AUROC internal 0.75 , AUROC external ranging from 0.63 to 0.79 ) had good predictive validity [Table 1]. Discrimination for all other outcomes was lower, with al $A U C<0.7$ in internal validation. For detailed results see: https://data.ohdsi. org/ehdenRaPrediction/

Table 1. Internal (Optum) and external validation results: AUC ROC for discrimination

\begin{tabular}{lccc}
\hline Database & Acute MI within 2y & Stroke within 2y & Serious Infection within 3m \\
\hline Optum (internal) & 0.77 & 0.78 & 0.75 \\
PanTher & 0.76 & 0.78 & 0.74 \\
IQVIA_AMBEMR & 0.76 & 0.72 & 0.66 \\
CCAE & 0.73 & 0.73 & \\
IQVIA_GERMANY & 0.64 & 0.70 & 0.67 \\
IQVIA_THIN & 0.62 & 0.65 & 0.61 \\
MDCR & 0.68 & 0.68 & 0.63 \\
IQVIA_HOSPITAL & 0.67 & 0.63 & 0.71 \\
MDCD & 0.72 & 0.79 & 0.82 \\
JMDC & 0.49 & 0.75 & \\
IQVIA_LPDFRANCE & 0.69 & & \\
Estonia & 0.67 & 0.77 & \\
IQVIA_AUS & 0.58 & & \\
IPCI & 0.68 & & \\
SIDIAP & 0.65 & 0.75 & \\
\end{tabular}

Conclusion: Clinical tools were developed that successfully identify subjects at risk of MI, stroke and serious infection at the initiation of first-line MTX therapy The developed algorithms had good transportability and generally, the models with high AUROC had adequate internal calibration although some external validations show they could benefit from recalibration. For short-term opportunistic and all infections, as well as 5-year cancer models, we were unable to achieve a high enough AUROC to warrant validating externally.

Disclosure of Interests: Cynthia Yang: None declared, Ross Williams: None declared, Joel Swerdel Shareholder of: J\&J shares, Grant/research support from: Full-time employment salary from Janssen, Consultant of: Janssen employee, Employee of: Janssen, Paid instructor for: Janssen employee, have instructed at conferences, Speakers bureau: Janssen employee, have spoken at conferences, Meghna Jani Speakers bureau: Grifols, Talita Duarte-Salles: None declared, Katerina Chatzidionysiou Consultant of: AbbVie, Pfizer, Lilly., Daniel Prieto-Alhambra Grant/research support from: Professor Prieto-Alhambra has received research Grants from AMGEN, UCB Biopharma and Les Laboratoires Servier, Consultant of: DPA's department has received fees for consultancy services from UCB Biopharma, Speakers bureau: DPA's department has received fees for speaker and advisory board membership services from Amgen, Patrick Ryan: None declared, Peter Rijnbeek: None declared

DOI: 10.1136/annrheumdis-2020-eular.3606

\begin{tabular}{|l|l|}
\hline OP0217 & INVOLVEMENT OF LARGE JOINTS AT DISEASE \\
& PRESENTATION IS ASSOCIATED WITH DIVERSE \\
& HISTOPATHOLOGICAL FEATURES AND CLINICAL \\
OUTCOMES IN EARLY RHEUMATOID ARTHRITIS
\end{tabular}

F. Rivellese ${ }^{1}$, F. Humby ${ }^{1}$, G. Lliso Ribera ${ }^{1}$, A. Nerviani ${ }^{1}$, E. Sciacca ${ }^{1}$, G. Giorli ${ }^{1}$, R. Hands ${ }^{1}$, L. Fossati-Jimack ${ }^{1}$, G. Thorborn ${ }^{1}$, M. Lewis ${ }^{1}$, C. Pitzalis ${ }^{1}$. ${ }^{1}$ Queen Mary University of London, Centre for Experimental Medicine and Rheumatology, London, United Kingdom

Background: The involvement of large joints at disease presentation in early Rheumatoid Arthritis (RA) has been associated with severe disease activity. A the same time, the clinical heterogeneity of RA is known to be mirrored by heterogeneity of synovial inflammation, with specific histological patterns (pathotypes) 